\title{
A Statistical Model for Estimating Provision for Doubtful Debts
}

\author{
Dhruba Kumar Budhathoki
}

\begin{abstract}
This paper attempts to analyze a statistical model for estimating provision for doubtful debts based on available data and literature. Where a significant portion of an enterprise's assets are tied up in Sundry Debtors (of any kind), the accuracy achieved in estimating this provision for doubtful debts assumes a special significance. This is very much well known to anyone who is seriously connected with financial matters, whether under any statute or for carrying out management accounting exercise. However, this paper is not intended to over-emphasize this aspect of financial management accounting. The purpose of this paper is to attempt to build a statistical model to enhance the degree of accuracy in this regard. The customary practice prevalent, at least in Nepal, is to estimate it mainly by applying a highly subjective judgmental process, bordering on a hunch-oriented process.
\end{abstract}

Keywords: Conditional probabilities, iteration, markov chain, realisation, steady state, stochastic process, transition matrix.

\section{Introduction}

A portion of the total debtors appear in the books at the close of the year is doubtful. Those debtors are almost irrecoverable on account of their poor financial condition, insolvency, dishonesty, lunacy and death. Though, at present the exact amount of such irrecoverable debtors are not known the amount can be estimated on the basis of proper assessment of all the debtors and their past accounting records. Hence, estimation for such anticipated irrecoverable debts should be made for accounting purpose, it is the estimated amount set aside to meet anticipated loss, an account of irrecovery of the debts. However, to achieve some perceived degree of objective accuracy, some enterprises follow a two-step process.

First, the debtor's accounts are classified into age categories, which reflect the stage of account delinquency, that is current accounts, accounts one month past due, accounts two months past due, and so forth. If the enterprise has a large number of accounts, the aging has been customarily performed on a sample basis and the frequency distribution of money, grouped according to age category, estimated from the sample for the total universe of accounts.

The second step in estimating the allowance (provision) for doubtful accounts involves the application of 'loss expectancy' rates to the monetary amount in each age group of the frequency distribution obtained by the sampling process. The loss expectancy rates are judgment estimates of the proportion of money in each age category liable (likely) to become uncollectable. In a sense, these loss expectancy rates are 'policy parameters', for they are not only based on past experience but are also functions of such things as the enterprise's expectations of economic conditions, its conservatism in accounting policy and other similar factors. 
As a continuation of research, the second-step was a logical area for investigation. While it did not seem likely that all of the judgment factors involved in setting of loss expectancy rates could be eliminated, it did appear feasible to develop a scientific approach to determine these rates (Davidson, 1968). Accordingly, research into this problem was initiated. This paper discusses a method which has been developed and applied successfully also by (Murali, 1994). In addition, some of the managerial implications of the method are also discussed.

\section{Data and Methods}

The method used in this paper is based on 'Markov Analysis' (Kemeny, 1960). Technically, it belongs to the Theory of Probability and makes profuse use of vectors and Matrices. It is, therefore, essential that the reader acquire clear understanding of the related mathematical concepts and the tools applied. At the end of the paper, the list of 'Further Readings', is meant for providing guidance to the uninitiated in this respect.

It is useful to gain an insight into terms, concepts and techniques of Markov Chain for being able to follow the theme of the discussion contained in this paper.

Markov Chain : A 'Markovian Process' is, therefore, a stochastic series of independent and progressive changes, that is, a process in which the probability of a transition form one state to another depends only upon the present state of the process. It has following properties:

a. The outcome of each experiment is one of a finite number of possible outcomes: $a_{1} a_{2}, \ldots a_{1}$

b. It is assumed that the probability of outcome aj of any given experiment is not necessarily independent of the outcomes of previous experiments, but depends at most upon the outcome of the immediately preceding experiment.

c. It is assumed that there are given numbers of pij which represent the probability of outcome a occurred on the previous experiment.

i) The outcomes $a_{1}, a_{2} \ldots \ldots$ a are called 'States'.

ii) The numbers pij are called "Transition Probabilities".

d. If it is assumed that the process begins in some particular state, then there is enough information to determine the decision treemeasure of the process and calculate probabilities of statements relating to the overall sequence of experiments. A process of this kind is called a Markov Chain Process'. [Sec. (f) below]

Notes: (i) A process is a series of independent and progressive changes (in state, here).

(ii) When the Probability Theory is applicable to a process, then it is called a 'Random' on 'Stochastic Process'.

e. A 'Markovian Chain Process', it is observed from the above statements, is a Markovian Process in which a stationary transition matrix applies to every change of state.

An 'Array' or a 'Matrix' when it indicates the 'Conditional Probabilities' of a process which is changing from one state to other possible states, including the same state, is called a 'Transitional Matrix'.

Two events A and B are said to be dependent when B can occur only when A is known to have occurred or vice-versa. The probabilities associated with such events are called 'Conditional Probabilities.'

g. A condition of equilibrium in which further changes do not change the probabilities of finding the process in various states is called a 'Steady State'. 


\section{Presentation and Analysis}

From the methodology stated above, it can be concluded that a Markov Process is such a process where the occurrence of future state depends on and only on the immediately preceding state. Such a kind of dependence is expressed as a 'Transition Matrix' indicating an intermediate state. In other words, Transition Matrix relates the former state to the latter state. This explains the behavior of the various constituents of a specific system. The relation between every particular state and the earlier one is explained by the Transition Matrix.

Testing applicability of Markov Process Rules:

It can be examined the following Matrix to see if Markov's Rule is applicable to it or not.

Table I: Application of Markov Rule

\begin{tabular}{lccccc}
\hline \multicolumn{1}{c}{ State } & I & II & III & IV & V \\
\hline Original Age Category & $31-60$ (days) & $61-105$ (days) & $106-182$ (days) & Realised & Bad debts \\
\hline I 31-60 & 0 & $40 \%$ & $10 \%$ & $50 \%$ & 0 \\
II 61-105 & 0 & 0 & $60 \%$ & $40 \%$ & 0 \\
III 106-128 & 0 & 0 & 0 & $30 \%$ & $70 \%$ \\
IV Realised & 0 & 0 & 0 & $100 \%$ & 0 \\
V Bad Debt & 0 & 0 & 0 & 0 & $100 \%$ \\
\hline
\end{tabular}

Source: Adopted from Gupta, 1995

Table II: Matrix Form

\begin{tabular}{lccccc}
\hline \multicolumn{1}{r}{ State } & I & II & III & IV & V \\
\hline & $31-60$ & $61-105$ & $106-182$ & Realised & Bad debts \\
\hline I 31-60 & 0 & 0.4 & 0.1 & 0.5 & 0 \\
II 61-105 & 0 & 0 & 0.6 & 0.4 & 0.0 \\
III 106-182 & 0 & 0 & 0.0 & 0.3 & 0.7 \\
IV Realised & 0 & 0 & 0.0 & 1.0 & 0.0 \\
V Bad Debt & 0 & 0 & 0.0 & 0.0 & 1.0 \\
\hline
\end{tabular}

Source : Adopted from Gupta, 1995

Markov Chain Analysis : It is a method that is concerned with predicting the future behaviour of a variable by analyzing the current behaviour of that particular variable.

If an enterprise is in a position to assess the behaviour of its debtors accounts in the past, then Markov Theory could be applied to estimate the amount of 'bad debts' at the end of the period or for a set of sales, provided Markov's Theory was found to be applicable to the behaviour of the debtors accounts.

i) Notice that in the above matrix $\bar{A}$, the sum of the elements of each Row is 1 (for row $1: 0.0+0.4+0.1+0.5+0.0=1.0$ ). This must be so in any Transitional Matrix (matrix of transitional probabilities), since the elements of the i-th row represent the probabilities for all the possibilities when the process is in state ai.

ii) The above kind of Matrix $\bar{A}$ is called the 'Initial Matrix', as mentioned at the top left corner of the table. 
Markovian Interpretation of Table I : table I exhibits five states (marked I, II, III, IV and IV) and the actual collection pattern of debtors' accounts of an assumed enterprise. It indicates that out of the 31-60 days, (dues, $40 \%$ is realised in the next period of 61 to 105 days; $10 \%$ is realised in the 106-182 day period and 50\% has been collected in the 31-60 day) - period itself.

Similarly, out of the 61-105 days old outstanding, 60\% are collected during the 106-182 day-period while $40 \%$ are collected during the stated period of 61-105 days. Out of the 106182 days old outstanding 30\% could be realized during the period itself (106-182 days) and $70 \%$ turned out to be bad. State IV marked 'Realised' indicates those debts which have been collected in due time. State V marked 'Bad Debts' indicates ab inilio bad debts.

In practice, some accounts are repaid even after reaching the 'bad debt' category. This, however, does not materially affect the realistic nature of the model. First, the bad debt category may be selected so that the prospect of subsequent recovery is very small, second, the model treatment corresponds to common accounting treatment, that is, accounts are 'written off on reaching a specific age category which is over 182 days (six months) for the model under discussion and recoveries are treated as separate transactions.

Thus, it is observed from the above discussion that the Matrix $\bar{A}$ under consideration follows the Markovian process as the probability of a transition from one state to another state depends upon the present state. For example the dues between (say) 61 to 105 days, depend upon the current state of outstanding for the 31-60 day period. Again, it is a Markovian Chain since the transition (change) into the subsequent states depends upon fixed probabilities. The concept of fixed probabilities is clear to the reader when one follows the workings related to the model developed in this connection. This involves a 'Transition Matrix (already explained) and a Steady state Matrix' (already explained), as can be seen later on. The Markovian process/ chain Method Used in the Model for Estimating Provision for Doubtful Debts:

Mathematicians have developed a number of theorems based on Markov Chain/Process/ Concepts for many purposes, including our present problem of estimating more objectively and accurately the amount of 'doubtful debt' provision. The one (theorem) which it can be made use of in the present context is being used successfully (Murali, 1994), is described below. At the outset it is quite helpful if it knows that it calls a process a 'Repititive Markov Chain'. When it starts off a Markov Chain a number of times each period and follow all of these until absorption, that is, attainment of a 'steady state'.

Table III: Initial Transition and steady state matrices

\begin{tabular}{lccccc}
\hline \multicolumn{1}{c}{ State } & I & II & III & IV & V \\
\hline $31-60$ (I) & $31-60(\mathrm{I})$ & $61-105(\mathrm{II})$ & $106-182(\mathrm{III})$ & Realised (IV) & Bad debts (V) \\
61-105 (II) & 0 & 0.4 & 0.1 & 0.5 & 0 \\
106-128 (III) & 0 & 0 & 0.6 & 0.4 & 0.0 \\
Realised (IV) & 0 & 0 & 0.0 & 0.3 & 0.7 \\
Bad Debt (V) & 0 & 0 & 0.0 & 1.0 & 0.0 \\
\hline
\end{tabular}

Source : Adopted from Gupta, 1995 
At the same time, it is also necessary to have a clear insight into one more mathematical operation (unless the reader is already familiar with it), namely, the ;'Iterative process'.

Iterate: To iterate means to repeat. So is an 'interacted differential, since $\mathrm{Y}$ is differentiated three times.

Iterative: An iterative process involves repetition of a sequence to improve some result.

It has been given below figure I, which illustrates an iterative way of finding the result of $\sqrt[3]{10}$

It can be applied the following Theorem (process/method) based on Markov Chain concepts. To compute precisely, when all the debtor's accounts are terminated in two categories, namely, 'Realised' and 'Bad Debts' (this is a Steady Statecondition), the Initial Matrix shown in Table II is multiplied by itself several times until the Steady State Matrix is obtained. Any further multiplication is only repeat the same steady matrix.

It happens this way because of the mathematical property of an 'Upper level Triangular Matrix'. This means, there are non-zero elements only above the principal diagonal of a square matrix (with only zero values below). A close look at the broken line (-----) representing the principal diagonal Matrix in Table II illustrates the point.

Illustration with Notations:

\begin{tabular}{cccccc}
$\mathrm{T}$ & $\overline{\mathrm{A}}$ & $=$ & $\overline{\mathrm{A}}$ & $\mathrm{X}$ & $\overline{\mathrm{A}}$ \\
$\mathrm{T}_{2}$ & $\overline{\mathrm{A}}$ & $=$ & $\mathrm{T}_{1}$ & $\overline{\mathrm{A}}$ & $\overline{\mathrm{A}}$ \\
$\ldots \ldots \ldots \ldots \ldots \ldots \ldots \ldots \ldots \ldots \ldots$. & \\
\cline { 1 - 2 } & $\mathrm{Tn}$ & $\overline{\mathrm{A}}$ & $=$ & $\mathrm{T}_{\mathrm{n}-1} \overline{\mathrm{A}}$ & $\mathrm{X} \overline{\mathrm{A}}$
\end{tabular}

This is a steady state matrix's general formula.

For the data incorporated in Table III above, the steady state Matrix is obtained at $\mathrm{T}_{2} \overline{\mathrm{A}}$; since Iteration 3 repeats the result of Iteration 2. In other worlds, in the given example, steady state is achieved at $\mathrm{T}_{2} \overline{\mathrm{A}}$. Hence, here $\mathrm{n}=2$.

Iterpretation of Markovian Steady State Matrix (Ts $\bar{A})$ :

Ts $\bar{A}$ shows that part of the total debtors' accounts which could be ultimately realized. This is given by the 'Realised' (R) column of the Ts $\bar{A}$, as follows:

Age Category Realised (R) Percentage (\%)
a) $\quad 31-60$ days
$0.762=76.2$
This $76.2 \%$ is the recovery rate of the initial dues in this age category.
Age category realized (R) Percentage (\%)
b. $\quad 61-105$ days $\quad 0.580=58.0 \%$
This $58.0 \%$ is the recovery rate of the initial dues in this age category.
c. $\quad 06-182$ days $\quad 0.300=30.0 \%$

This $30.0 \%$ is the recovery rate of the initial dues in this age category. Illustrative Example : Suppose, a large company has posted a sales figure of Rs. 1,500 lakhs; out of which Rs 300 lakhs is realized within 0-30 days from the date of sale. Management expectation of collection of the remaining Rs 1,200 lakhs is depicted below: 
Spread Out collection Period (in days)

$\begin{array}{llll}31-60 & 61-105 & 106-182 & \text { Total (Rs Lakhs) } \\ 500 & 600 & 100 & 1.200\end{array}$

But, the management account/auditor can make the following estimates on the basis of the Markov Steady State Matrix developed in this paper, assuming that it holds good for the company under review, on the basis of its past information.

Table IV : Iteration 1: $(=\overline{\mathrm{A}} \mathrm{X} \overline{\mathrm{A}})=\left(\mathrm{T}_{1} \overline{\mathrm{A}}\right)$

\begin{tabular}{lccccc}
\hline \multicolumn{1}{c}{ State } & $31-60(\mathrm{I})$ & $61-105(\mathrm{II})$ & 106-182 (III) & Realised (IV) & Bad debts (V) \\
\hline 31-60 (I) & 0 & 0 & 0.240 & 0.690 & 0.070 \\
61-105 (II) & 0 & 0 & 0.000 & 0.580 & 0.420 \\
106-128 (III) & 0 & 0 & 0.000 & 0.300 & 0.700 \\
Realised (IV) & 0 & 0 & 0.000 & 1.00 & 0.000 \\
Bad Debt (V) & 0 & 0 & 0.000 & 0.000 & 1.000 \\
31-60 (I) & 0 & 0 & 0 & 0.762 & 0.238 \\
61-105 (II) & 0 & 0 & 0 & 0.580 & 0.420 \\
106-128 (III) & 0 & 0 & 0 & 0.300 & 0.700 \\
Realised (IV) & 0 & 0 & 0 & 1.000 & 0.000 \\
Bad Debt (V) & 0 & 0 & 0 & 0.000 & 1.000 \\
\hline
\end{tabular}

Source : Adopted from Gupta, 1995

Table V: Iteration 2: $(\overline{\mathrm{A}} \times \overline{\mathrm{A}} \times \overline{\mathrm{A}})=\left(\mathrm{T}_{1} \overline{\mathrm{A}} \times \overline{\mathrm{A}}\right)=\left(\mathrm{T}_{2} \overline{\mathrm{A}}\right)$

\begin{tabular}{cccccc}
\hline \multirow{2}{*}{$\begin{array}{c}\text { Collection Period } \\
\text { (in days) }\end{array}$} & $\begin{array}{c}\text { Initial Dues (Rs. } \\
\text { In lakhs) }\end{array}$ & \multicolumn{2}{c}{ Realisation } & \multicolumn{2}{c}{ Bad debts } \\
\cline { 3 - 6 } & & Amount & $\%$ & Amount & $\%$ \\
\hline $31-60$ & 500 & 381 & 76.2 & 119 & 23.8 \\
$61-105$ & 600 & 348 & 58.0 & 252 & 42.0 \\
$106-128$ & 100 & 30 & 30.0 & 70 & 70.0 \\
Total & 1,200 & 759 & & 441 & \\
\hline
\end{tabular}

Source : Adopted from Gupta, 1995

The above computation calls for a provision of Rs. 441 lakhs for doubtful debts (as against the management's 'Hench-based' 10\%, that is Rs. 120 lakhs, perhaps). Shouldn't then the auditor of a large company, say a bank, heave a sigh of relief and be silently thankful to the Russian mathematician, Andrei A. Markov?

Computer Aid: Experts recommend the use of Quattro Pro, Spread Sheets Softwares like, Lotus 1-2-3, Supercale, etc for carrying out the matrix multiplications through/ DMM command. Incidentally, it has, however been, used the Basic Language Programme. 


\section{Conclusion}

From what has been discussed above, it may be summed up by stating that given a matrix of transition probabilities $\mathrm{P}$ and also given a vector of sales (including new sales), either constant or variable by period (not dealt with in this model), the following results are obtained:

- Estimated loss expectancy rates by age category.

- Estimated Provision for Doubtful Debts.

- The steady state age distribution of debtors' accounts.

- Variances of the above results in (2) and (3) - not dealt with here, but can be dealt with through some sophisticated tools (let the reader try it out).

- Generalisation of the above results to the cyclical case not discussed here. But, can be tackled with some sophisticated techniques.

It has already referred to this assumption, though it is not true in the real-world. There is cyclical change in sales and/or in transition probabilities. This validity of assumption depends on the magnitude of these changes. However, researches are on in this regard. Perhaps, soon it is possible, to predict the changes in transition probabilities in a much more objective manner and with greater accuracy.

Initially, the research was aimed at estimating loss expectancy rates, which has now gratifyingly gone much beyond it. At present, it seems to be a generally accepted belief that the Markov Chain/Transition Probability description of the behavior of debtors' accounts provides a valuable insight into the search for better methods of managing debtors' accounts. Management of debtors in most enterprises continually pose credit-policy problems. To aid management in this decision making area, a variety of statistics are used to reflect current economic conditions and the overall status of the debtors' accounts, such as various ratios. All of these measures have some value as indices of current behavior of debtors' balances/ accounts. None, however, seems to offer the comprehensive picture of such behavior that is provided by the matrix of transition probability and related model outcomes. With further advanced research in this field, managers, management accountants and auditors may hopefully look forward to more effective and efficient models to emerge to come to their aid in managing on of the most important financial assets.

\section{BIBLIOGRAPHY}

David S. C. (1968). Estimation of the allowance for doubtful accounts. England: Penguin Books Ltd,.

Gupta, A. M. (1995). Estimating provision for doubtful debts.The Journal of The Management Accountant, 30(5), 378-383.

Murali, R. S. (1994). Markov analysis: Dealing with bad debts. The Journal of Management Accountant 30 (5), 365-372.

Nostrand, V. A., \& Kemeny, S. (1960). Finite markov chains. The Journal of the Management Accountant, 30 (8), 594-598.

Thomption; K. (1985). Introduction to finite mathematics. New Delhi: Prentice Hall of India Pvt. Ltd. 